\title{
A new solution to the solar neutrino deficit
}

\author{
Alexander Friedland ${ }^{1}$ and Andrei Gruzinov ${ }^{2}$ \\ ${ }^{1}$ School of Natural Sciences, Institute for Advanced Study, Princeton NJ, USA 08540 \\ ${ }^{2}$ New York University, 4 Washington Place, New York, NY 10003
}

(November 27, 2002)

\begin{abstract}
We reexamine the transition magnetic moment solution to the solar neutrino problem. We argue that the absence of large time variations in the Super-Kamiokande rate provides strong evidence against spin-flavor flip in the solar convective zone. Spin-flavor flip could, however, occur in the primordial magnetic field in the radiative zone. We compute the longest lived toroidal mode for this field and show that spin-flavor flip in the radiative zone can account for all available solar data.
\end{abstract}

\section{INTRODUCTION}

In recent years, the progress in the field of solar neutrino physics has been remarkable. This progress culminated when the SNO collaboration conclusively demonstrated the presence of a non-electron neutrino component in the flux of solar neutrinos [1,2].

The SNO experiment measured the flux of the ${ }^{8} \mathrm{~B}$ solar neutrinos using three different reactions: (i) the charged current (CC) process $\nu_{e}+d \rightarrow p+p+e^{-}$, (ii) the elastic scattering (ES) process $\nu_{x}\left(\bar{\nu}_{x}\right)+e^{-} \rightarrow \nu_{x}\left(\bar{\nu}_{x}\right)+e^{-}$, and (iii) the neutral current (NC) process $\nu_{x}\left(\bar{\nu}_{x}\right)+d \rightarrow$ $p+n+\nu_{x}\left(\bar{\nu}_{x}\right)$. A high statistics ES measurement, in the same energy range, had also been previously carried out by the Super-Kamiokande (SK) experiment [3]. While the CC process selects only electron neutrinos, the other two processes are sensitive to neutrinos as well as antineutrinos of all active flavors.

The results of the measurements are as follows $[2,4]:{ }^{*}$

$$
\begin{aligned}
R_{\mathrm{SNO}}^{\mathrm{CC}} & =1.76 \pm 0.11 \times 10^{6} \mathrm{~cm}^{-2} \mathrm{~s}^{-1}, \\
R_{\mathrm{SNO}}^{\mathrm{ES}} & =2.39 \pm 0.27 \times 10^{6} \mathrm{~cm}^{-2} \mathrm{~s}^{-1}, \\
R_{\mathrm{SK}}^{\mathrm{ES}} & =2.35 \pm 0.08 \times 10^{6} \mathrm{~cm}^{-2} \mathrm{~s}^{-1}, \\
R_{\mathrm{SNO}}^{\mathrm{NC}} & =5.09 \pm 0.63 \times 10^{6} \mathrm{~cm}^{-2} \mathrm{~s}^{-1}, \\
R_{\mathrm{SNO}}^{\mathrm{NC} 2} & =6.42 \pm 1.67 \times 10^{6} \mathrm{~cm}^{-2} \mathrm{~s}^{-1} .
\end{aligned}
$$

They are impossible to reconcile assuming the incident flux consists entirely of electron neutrinos $[1,5,2]$, and hence some other particles must be contributing to the ES and NC event rates. Chronologically, the first detector to observe events caused by these other particles was SK, but it was not possible to separate these events from the $\nu_{e}$-induced events until the SNO CC data became available. One can therefore say that the SNO measurement has effectively reclassified SuperKamiokande as an appearance experiment.

The interpretation of the SNO results, however, is not unambiguous. While the "excess" NC and ES events are

\footnotetext{
${ }^{*} R_{\mathrm{SNO}}^{\mathrm{NC} 1}$ was found assuming undistorted ${ }^{8} \mathrm{~B}$ neutrino energy spectrum, while $R_{\mathrm{SNO}}^{\mathrm{NC} 2}$ was found when this assumption was relaxed.
}

most often ascribed to muon (and tau) neutrinos, they can be also caused by muon (and tau) antineutrinos. The antineutrinos in question must be of the muon or tau types, since electron antineutrinos would be easily identified through the reaction $\bar{\nu}_{e}+p \rightarrow n+e^{+}[6]$. Both $\nu_{\mu, \tau}$ and $\bar{\nu}_{\mu, \tau}$ scatter on electrons and deuterium nuclei through their neutral current (NC) interactions, with similar cross sections. At $E_{\nu} \sim 10 \mathrm{MeV}$,

$$
\int_{T_{\min }}^{T_{\max }} d T_{e} \frac{d \sigma\left(\bar{\nu}_{\mu, \tau} e^{-}\right)}{d T_{e}} / \int_{T_{\min }}^{T_{\max }} d T_{e} \frac{d \sigma\left(\nu_{\mu, \tau} e^{-}\right)}{d T_{e}} \sim 0.8
$$

where the integral over the electron recoil energy $T_{e}$ ranges from $T_{\min }=5 \mathrm{MeV}$ to $T_{\max }=E_{\nu} /\left(1+m_{e} / 2 E_{\nu}\right)$ $\mathrm{MeV}$. Similarly, using the latest available calculations of the $\nu_{x}\left(\bar{\nu}_{x}\right)+d \rightarrow p+n+\nu_{x}\left(\bar{\nu}_{x}\right)$ cross sections [7] and integrating over the undistorted ${ }^{8} \mathrm{~B}$ reaction spectrum, $f\left(E_{\nu}\right),[8]$, one finds

$$
\int d E_{\nu} f\left(E_{\nu}\right) \sigma\left(\bar{\nu}_{\mu, \tau} d\right) / \int d E_{\nu} f\left(E_{\nu}\right) \sigma\left(\nu_{\mu, \tau} d\right) \sim 0.95
$$

Assuming the $\nu_{e} \rightarrow \nu_{\mu, \tau}$ conversion mode, Eqs. (1-4) imply that the electron neutrino survival probability is $P_{e e} \simeq 0.35 \pm 0.04\left(P_{e e} \simeq 0.27 \pm 0.06\right.$ if Eqs. $(1-3,5)$ are used). If instead the $\nu_{e} \rightarrow \bar{\nu}_{\mu, \tau}$ mode is assumed, the same data imply a somewhat higher flux of all active neutrinos, namely, $\Phi_{\mathrm{tot}}=5.25 \pm 0.66 \mathrm{~cm}^{-2} \mathrm{~s}^{-1}$ if one uses Eqs. (1-4) or $\Phi_{\text {tot }}=6.64 \pm 1.75 \mathrm{~cm}^{-2} \mathrm{~s}^{-1}$ if one uses Eqs. (1-3,5). The corresponding survival probabilities are $P_{e e} \simeq 0.34 \pm 0.05$ or $P_{e e} \simeq 0.27 \pm 0.07$. Thus, both conversion modes are capable of accounting for the measured event rates. Moreover, both yield the total flux that is consistent with the Standard Solar Model (SSM), which predicts $5.05_{-0.8}^{+1.0} \times 10^{6} \mathrm{~cm}^{-2} \mathrm{~s}^{-1}[9]$ or $5.93 \pm 0.89 \times 10^{6}$ $\mathrm{cm}^{-2} \mathrm{~s}^{-1}[10]$, depending on the input value of the S17 parameter used. Therefore, in interpreting the experimental data both possibilities should be considered.

The $\nu_{e} \rightarrow \nu_{\mu, \tau}$ conversion is predicted in the flavor oscillation scenario [11], while $\nu_{e} \rightarrow \bar{\nu}_{\mu, \tau}$ is predicted in the neutrino spin-flavor flip (SFF) scenario [12-16]. The flavor oscillation scenario has been extensively studied over the years. The goal of this paper is to reexamine the SFF scenario and to point out the existence of a new solution. 
In the SFF scenario, the neutrino is postulated to couple to the electromagnetic field through a magnetic moment, $\mu$, interaction. A neutrino propagating in the solar magnetic field undergoes a spin rotation, and, if it is a Majorana fermion, becomes an antineutrino, in the process also changing its flavor.

Majorana fermions are most naturally described using the two-component Weyl spinor notation. The coupling in question is given by a Lagrangian term

$$
\mathcal{L}_{E M}=-\frac{1}{2} \mu_{a b}\left(\nu^{\alpha}\right)_{a}\left(\sigma^{\mu \nu}\right)_{\alpha}^{\beta}\left(\nu_{\beta}\right)_{b} F_{\mu \nu}+\text { h.c. },
$$

where $\left(\sigma^{\mu \nu}\right)_{\alpha}{ }^{\beta} \equiv\left(\sigma_{\alpha \dot{\alpha}}^{\mu} \bar{\sigma}^{\dot{\alpha} \beta \nu}-\sigma_{\alpha \dot{\alpha}}^{\nu} \bar{\sigma}^{\dot{\alpha} \beta \mu}\right) / 2, \sigma_{\alpha \dot{\alpha}}^{\mu} \equiv(1, \vec{\sigma})$, $\bar{\sigma}^{\dot{\alpha} \beta \nu} \equiv(1,-\vec{\sigma}) . \quad \mu$ and $\nu$ are Lorentz indices, $\alpha$ and $\beta$ are Weyl spinor indices, and $a$ and $b$ are the flavor indices. Since the spinors anticommute, the $a=b$ terms in Eq. (8) vanish identically, and hence Majorana neutrinos cannot have magnetic moments [17]. They can, however, have transition $(a \neq b)$ moments which lead to a simultaneous spin and flavor change. This provides a natural scenario for $\nu_{e} \rightarrow \bar{\nu}_{\mu, \tau}$ transitions without also producing $\bar{\nu}_{e}$ states. Flavor mixing, if present, must be small to avoid a significant secondary $\bar{\nu}_{\mu, \tau} \rightarrow \bar{\nu}_{e}$ conversion. Regarding the naturalness of this assumption we note that out of the three mixing angles in the leptonic sector the one measured with atmospheric neutrinos is large and the one measured by reactor experiments is small, so that $a$ priori there is no natural value for the third angle.

In general, the evolution of the neutrino state is governed by a $6 \times 6$ Hamiltonian matrix. For simplicity, we consider a $4 \times 4$ case. In the basis $\left(\nu_{e}, \nu_{\mu}, \bar{\nu}_{e}, \bar{\nu}_{\mu}\right)$, the Hamiltonian is [15]

$$
H=\left(\begin{array}{cc}
H_{\nu} & \left(B_{x}-i B_{y}\right) M^{\dagger} \\
\left(B_{x}+i B_{y}\right) M & H_{\bar{\nu}}
\end{array}\right)
$$

where $B_{x, y}$ are the transverse components of the magnetic field and the $2 \times 2$ submatrices are given by

$$
\begin{aligned}
M & =\left(\begin{array}{cc}
0 & -\mu_{e \mu} \\
\mu_{e \mu} & 0
\end{array}\right), \\
H_{\nu} & =\left(\begin{array}{cc}
-\Delta \cos 2 \theta+A_{e} & \Delta \sin 2 \theta \\
\Delta \sin 2 \theta & \Delta \cos 2 \theta+A_{\mu}
\end{array}\right), \\
H_{\bar{\nu}} & =\left(\begin{array}{cc}
-\Delta \cos 2 \theta-A_{e} & \Delta \sin 2 \theta \\
\Delta \sin 2 \theta & \Delta \cos 2 \theta-A_{\mu}
\end{array}\right) .
\end{aligned}
$$

Here $\Delta \equiv \Delta m^{2} / 4 E_{\nu}, A_{e} \equiv \sqrt{2} G_{F}\left(n_{e}-n_{n} / 2\right)$ and $A_{\mu} \equiv \sqrt{2} G_{F}\left(-n_{n} / 2\right) . \Delta m^{2}$ is the neutrino mass-squared splitting, $E_{\nu}$ is its energy, and $n_{e}$ and $n_{n}$ are the electron and neutron number densities.

As will be described later, to obtain a significant $\nu_{e} \rightarrow \bar{\nu}_{\mu, \tau}$ conversion in the Sun requires $\mu_{e \mu} \sim\left(10^{-12}-\right.$ $\left.10^{-10}\right) \mu_{B}\left(\mu_{B} \equiv e / 2 m_{e}\right.$ is the Bohr magneton). The transition moment values in this range are below the direct laboratory bounds derived from the analyses of the $\nu+e \rightarrow \nu+e[18]$ and $\bar{\nu}+e \rightarrow \bar{\nu}+e$ [19] scattering data. Stronger bounds on the transition moment have been claimed from the analysis of various astrophysical processes, most notably from the study of the red giant populations in globular clusters [20]. The idea is that a sufficiently large transition moment would provide an additional cooling mechanism and change the red giant core mass at helium flash beyond what is observationally allowed. The bound stated in [20] is $\lesssim 3 \times 10^{-12} \mu_{B}$. Recently, there has been some disagreement between the stellar models and also a systematic shift to longer distances for clusters [21], and it would be very interesting to see an updated bound.

The compilation of various bounds on the size of the transition moment and the corresponding references can be found in [22].

\section{MAGNETIC FIELD IN THE CONVECTIVE ZONE}

The neutrino spin-flavor flip can occur either in the convective zone $(\mathrm{CZ})\left(0.71 R_{\odot} \lesssim r<R_{\odot}\right)$ or in the radiative zone (RZ) deeper in the solar interior. The two possibilities are physically quite different and require separate treatments. First, we consider the CZ case.

While the magnetic field in the inner part of the $\mathrm{CZ}$ cannot yet be measured directly, its basic properties can be inferred from the field at the solar surface, and it is widely believed that large scale field structures exist at some depth in the CZ [23]. Recent magnetohydrodynamic dynamo models argue that the field generation occurs in the shear layer near the bottom of the CZ. These models predict field values as large as $100 \mathrm{kG}$ [23], which significantly exceeds the turbulent equipartition value of $B \sim \rho^{1 / 6} L_{\odot}^{1 / 3} r^{-2 / 3} \sim 10^{4} \mathrm{G}^{\dagger}$. The helioseismological data provide an upper limit of $300 \mathrm{kG}$ on the magnitude of this field [24].

The field strength necessary for significant $\nu_{e} \rightarrow \bar{\nu}_{\mu, \tau}$ conversion can be estimated as $\mu_{e \mu} B \Delta l \sim 1$, where $\Delta l$ is the thickness of the magnetic field. One finds

$$
\left(\mu_{e \mu} / \mu_{B}\right)(B / 1 \mathrm{kG})\left(\Delta l / 0.1 R_{\odot}\right) \sim 5 \times 10^{-10}
$$

so that for $\mu_{e \mu} \sim 10^{-11} \mu_{B}$ and $\Delta l \sim 0.1 R_{\odot}$ the value $B \sim 50 \mathrm{kG}$ is required. Although the surface field, which reaches several $\mathrm{kG}$ in sunspots, is too weak to affect the evolution of the solar neutrinos, the field near the bottom of the $\mathrm{CZ}$ might have the right strength (provided $\left.\mu_{e \mu} \sim 10^{-11} \mu_{B}\right)$. This observation, and the natural expectation that the field in the interior of the $\mathrm{CZ}$ changes

\footnotetext{
${ }^{\dagger}$ This estimate is obtained by estimating the convective energy flux as $L_{\odot} \sim 4 \pi r^{2} v \rho v^{2} / 2$, where $v$ is the turbulent velocity, and assuming equipartition $\rho v^{2} / 2 \sim B^{2} / 8 \pi$.
} 
with the solar cycle, were the reasons the convective zone SFF were originally suggested as an explanation for an apparent anticorrelation between the Homestake event rate and the number of sunspots [13].

Over time, however, a paradigm shift has occurred. The idea of using this mechanism to explain variations of the neutrino rates was abandoned, especially in view of the SK data, which showed no seasonal or yearly variation, except for a hint of the expected $1 / r^{2}$ flux modulation. Instead, recent analyses [25] assume that the magnetic field in the $\mathrm{CZ}$ is somehow time independent, an assumption that we find untenable. The presence of a strong constant magnetic field inside the $\mathrm{CZ}$ would, on the one hand, almost certainly be revealed at the surface by convective mixing and, on the other hand, cause an asymmetry in the solar cycle. The strong toroidal field that is believed to exist in the overshoot layer, where convective mixing is small and the shear is large, should also be variable because it is generated from a variable radial field. It is therefore implausible that the SFF process in the $\mathrm{CZ}$ could cause a $\sim 70 \%$ depletion of the ${ }^{8} \mathrm{~B}$ neutrino flux, while producing no observable variations at SK during more than four years. This time span covers about half of the solar cycle, with an average sunspot number $\sim 10$ in 1996, and $\sim 120$ in 2000 [26].

In addition to the year-to-year variations, one also expects characteristic semiannual variations [13], for the following reason. The field on the surface of the Sun changes direction across the solar equator and vanishes at the equator (where indeed no sunspots are observed). The transition region, where the field is small, has the size $\sim 7 \times 10^{9} \mathrm{~cm}[13,27]$. The orbit of the Earth makes a $7^{\circ}$ angle with the plane of the solar equator and, since $95 \%$ of the ${ }^{8} \mathrm{~B}$ neutrinos are produced in the region $r<0.09 R_{\odot}[9]$, a significant fraction $(88 \%)$ of them passes through the transition region when the Earth crosses the plane of the solar equator. On the other hand, in March and September, when the Earth is farthest from the solar equatorial plane, this fraction is as low as $1 \%$. Thus, large semiannual variations are expected at Super-Kamiokande and are not observed [3].

We therefore regard the absence of variations in the SK data as strong evidence against SFF in the CZ.

\section{RELIC FIELD IN THE RZ}

We now turn to the possibility that the SFF process could occur in the radiative zone of the Sun. Unlike the convective zone, the radiative zone is not continuously mixed and rotates as a solid body. Therefore, it could in principle support a "frozen" magnetic field configuration.

The properties of magnetic fields in the solar interior were investigated by Cowling [28], and by Bahcall and Ulrich [29]. These authors consider poloidal magnetic fields. We have redone the calculation [30] using a modern solar

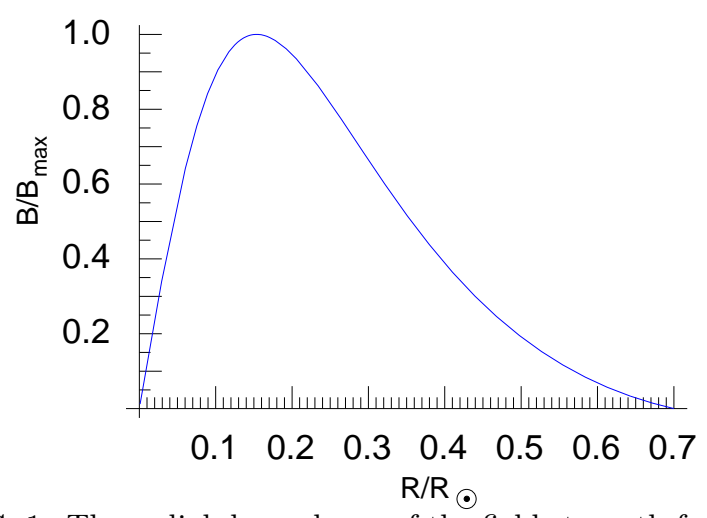

FIG. 1. The radial dependence of the field strength for the lowest toroidal mode in the RZ of the BP2000 SSM.

model (BP2000), and, more importantly, assuming that the field is toroidal. A strong poloidal field would penetrate the convective zone and would either dissipate over the solar lifetime, or be observable. We also assumed axisymmetry, which is physically plausible. Large asymmetry would lead to observable 27-day variations of the neutrino rate due to solar rotation.

For an axisymmetric toroidal field, the magnetic diffusion equation (describing Ohmic decay) was solved numerically. The eigenmodes are of the form $B=$ $e^{-t / \tau_{l, n}} F_{l, n}(r) P_{l}^{1}(\cos \Theta)$, where $l=1,2, . ., n=1,2, . .$, $P_{l}^{1}(\cos \Theta)$ is the associated Legendre polynomial and $F(r)$ is determined numerically. The $l=1, n=1$ mode has the longest lifetime, $\tau_{1,1}=24$ Gyr; its radial profile is shown in Fig. 1. For higher modes, $\tau_{2,1}=13 \mathrm{Gyr}$, $\tau_{1,2}=10 \mathrm{Gyr}, \ldots$ For comparison, the solar age is thought to be 4.6 Gyr [9]. Therefore, a toroidal field of complex spatial structure can exist in the RZ of the Sun.

Observations provide upper bounds on the allowed strength of this field [30]. Neutrino fluxes and helioseismological measurements of the sound speed both give $B \lesssim 10^{2}$ MG. Possible residual (non-rotational) oblateness and helioseismological measurements of the frequency splittings provide stronger bounds, $B \lesssim 7 \mathrm{MG}$ [30].

We next consider the neutrino evolution in the presence of this relic field and show that with appropriate parameter choice there exists a fit to all available solar neutrino data.

For simplicity, we assume no flavor mixing and that only the longest living eigenmode is present. This is sufficient to demonstrate the existence of the solution. The neutrino evolution equations then reduce to a pair of coupled equations,

$$
\begin{aligned}
i \partial_{l} \psi_{e} & =\left(A_{e}(r)-\Delta\right) \psi_{e}+\mu_{e \mu} B_{\perp}(l) \psi_{\bar{\mu}}, \\
i \partial_{l} \psi_{\bar{\mu}} & =\mu_{e \mu} B_{\perp}(l) \psi_{e}+\left(\Delta-A_{\mu}(r)\right) \psi_{\bar{\mu}} .
\end{aligned}
$$

Here $l$ is the distance along the neutrino trajectory and $B_{\perp}$ is the transverse component of the magnetic field. 


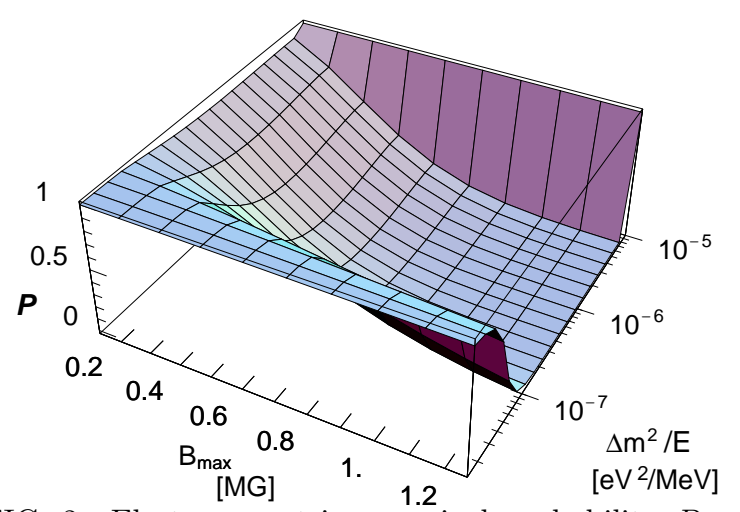

FIG. 2. Electron neutrino survival probability $P_{e e}$ as a function of $B_{\max }$ and $\Delta m^{2} / E_{\nu}$, for $\mu_{e \mu}=10^{-11} \mu_{B}$.

There are two free parameters, $\Delta m^{2}$ and the product of the neutrino magnetic moment $\mu_{e \mu}$ and the normalization of the magnetic field $B_{\max }$. For definitiveness, we set $\mu_{e \mu}=10^{-11} \mu_{B}$. Later we will address the possibility of having different values of $\mu_{e \mu}$.

Fig. 2 shows the survival probability $P_{e e}$ for electron neutrinos produced in the center of the Sun, as a function of $B_{\max }$ and $\Delta m^{2} / E_{\nu}$. The probability was obtained by solving the evolution equations (14) numerically on a grid of points.

The behavior of $P_{e e}$ can be given a simple analytical interpretation. The situation is completely analogous to the classical case of the small angle MSW effect $[15,14]$. The SFF process takes place in a thin layer around $r_{\text {res }}$ given by the condition $A_{e}\left(r_{\text {res }}\right)+A_{\mu}\left(r_{\text {res }}\right)=2 \Delta$. For $\Delta m^{2} / E_{\nu} \gtrsim 10^{-5} \mathrm{eV}^{2} / \mathrm{MeV}$, the resonance never occurs and therefore $P_{e e} \rightarrow 1$. For $\Delta m^{2} / E_{\nu} \lesssim 4 \times 10^{-8}$ $\mathrm{eV}^{2} / \mathrm{MeV}$, the resonance occurs close to the edge of the $\mathrm{RZ}$ where the field is small and hence once again $P_{e e} \rightarrow 1$ (or outside in the $\mathrm{CZ}$, in which case $P_{e e}$ varies with time). In the intermediate region, the conversion efficiency depends on the value of $\mu_{e \mu} B_{\perp}$ at $r=r_{\text {res }}$ : for $\mu_{e \mu} B_{\perp}$ $\gtrsim 0.7 \times 10^{-11} \mathrm{MG} \times \mu_{B}$ the conversion is adiabatic and $P_{e e} \rightarrow 0$.

Notice that for this description it is crucial that everywhere except in the thin resonance layer the off-diagonal term $\mu_{e \mu} B_{\perp}$ is much smaller than the diagonal splitting $\left(A_{e}(r)+A_{\mu}(r)\right) / 2-\Delta$. This allows one to ignore the fact that the off-diagonal coupling varies along the neutrino trajectory and use a small angle MSW formula for the level crossing probability

$$
P_{c}=\exp \left(-\left.\pi \frac{2\left(\mu_{e \mu} B_{\perp}(l)\right)^{2}}{\left|d\left(A_{e}+A_{\mu}\right) / d l\right|}\right|_{l=l_{\mathrm{res}}}\right) .
$$

The complications related to the large angle MSW effect [31] are likewise avoided. The neutrino survival probability is given by $P_{e e}=\left(1+\left(1-2 P_{c}\right) \cos 2 \theta_{\odot}\right) / 2$, where $\cos 2 \theta_{\odot}=\left(2 \Delta-A_{e}-A_{\mu}\right)\left[\left(2 \Delta-A_{e}-A_{\mu}\right)^{2}+\right.$ $\left.\left(2 \mu_{e \mu} B_{\perp}\right)^{2}\right]^{-1 / 2}$ is computed at the production point.

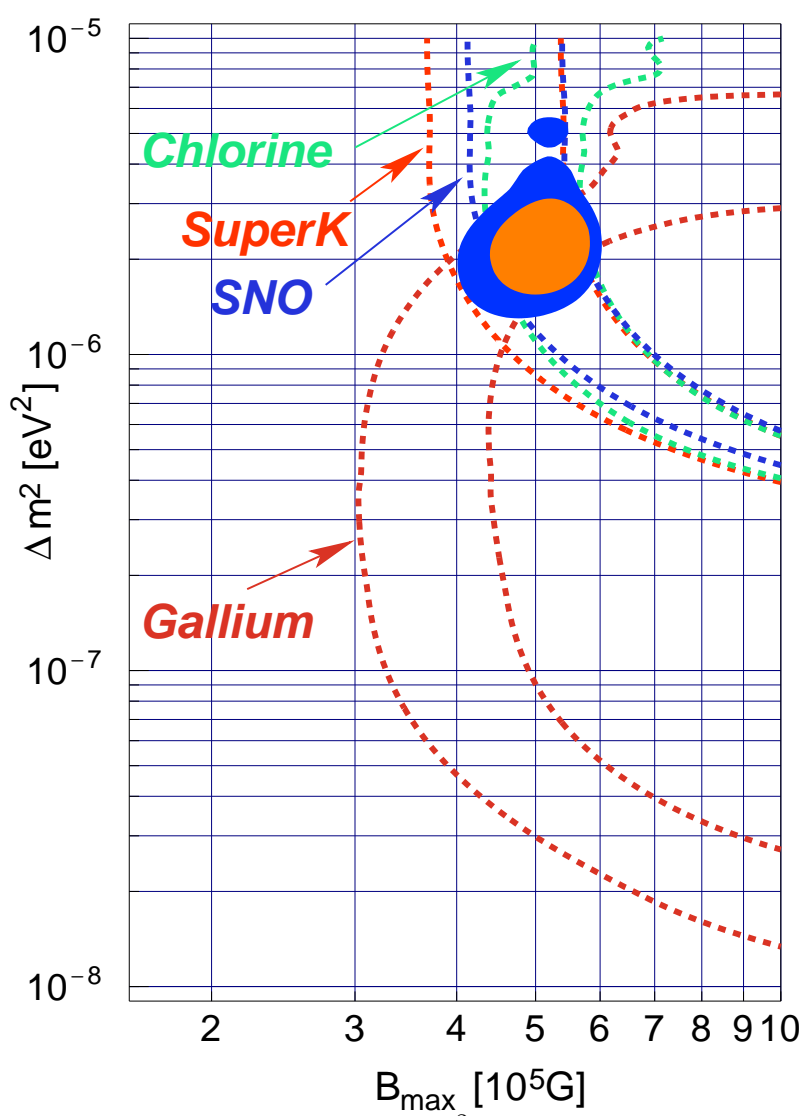

FIG. 3. Regions in the $\left(\Delta m^{2}, B_{\max }\right)$ parameter space where the rates of various solar neutrino experiments are reproduced.

We performed a fit to all available solar neutrino data. In our computations, we used the fluxes of the $p p,{ }^{7} \mathrm{Be}$, ${ }^{8} \mathrm{~B},{ }^{13} \mathrm{~N},{ }^{15} \mathrm{O}$, and pep neutrinos, as predicted by the BP2000 solar model [9]. The data to be fitted include the average rates of the GALLEX/GNO [32] and SAGE [33] experiments,

$$
R_{\mathrm{GA}}=74.7 \pm 5.1 \mathrm{SNU}
$$

the rate of the Homestake experiment [34],

$$
R_{\mathrm{CHL}}=2.56 \pm 0.23 \mathrm{SNU}
$$

the $\mathrm{CC}$ and $\mathrm{NC}$ rates of the $\mathrm{SNO}$ experiment as given in Eqs. $(1,4)$, and the rate and the energy spectrum of the SK experiment [3].

For a given production point in the solar core, the neutrino survival probability is determined according to Eqs. (14). To obtain the total flux of the neutrinos of a given energy, one needs to integrate over the neutrino production regions. The problem is technically quite nontrivial, because of the 3 -dimensional structure of the field and because only the field component transverse to the 


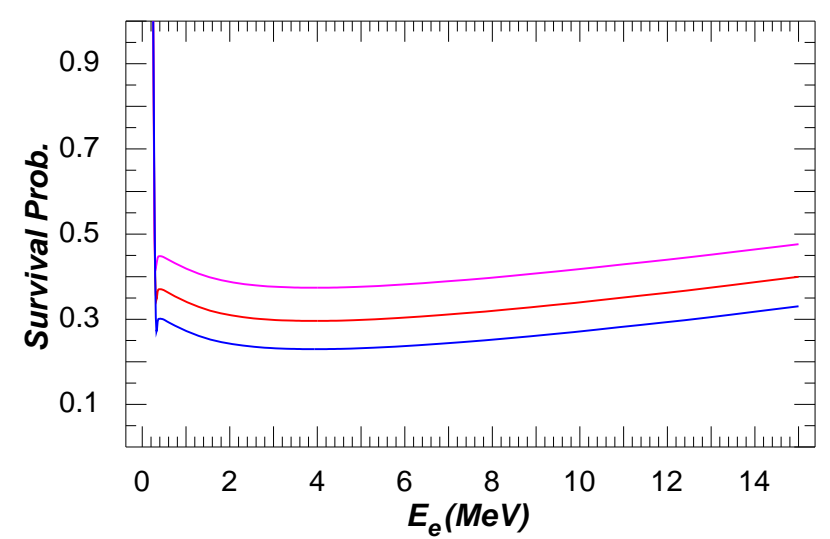

FIG. 4. Survival probabilities for an electron neutrino born in the center of the Sun for three values of $B_{\max }$ : $0.45 \mathrm{MG}$, $0.5 \mathrm{MG}$, and $0.55 \mathrm{MG}$ (top to bottom). $\Delta m^{2}=2.5 \times 10^{-6}$ $\mathrm{eV}^{2}$ was chosen.

neutrino trajectory enters the evolution equations. ${ }^{\ddagger}$ The correct integration is absolutely essential for computing the converted fraction of the low energy $p p$ neutrinos, which have a broad production region and for which the resonance occurs close to, and even inside, that region.

Fig. 3 shows regions of the parameter space where the event rates predicted for various experiments agree with the corresponding measured rates. The dashed lines delineate the bands where agreement is reached with a particular experiment. The intersection region, where a good fit to all rates is obtained, is characterized by $0.45 \mathrm{MG} \lesssim B_{\max } \lesssim 0.55 \mathrm{MG}$ and $1.5 \times 10^{-6}$ $\mathrm{eV}^{2} \lesssim \Delta m^{2} \lesssim 3 \times 10^{-6} \mathrm{eV}^{2}$. It represents our new solution to the solar neutrino deficit.

As an illustration, we list below our predictions for the point $\Delta m^{2}=2.5 \times 10^{-6} \mathrm{eV}^{2}$ and $B_{\max }=0.5 \mathrm{MG}$. We find that for this point the ${ }^{8} \mathrm{~B}$ flux is suppressed by a factor of 0.34 , the $p p$ flux - by a factor of 0.86 , and the ${ }^{7}$ Be flux - by a factor of 0.36 . The ${ }^{7} \mathrm{Be}$ suppression factor implies the effective Borexino flux suppression of 0.44, which is below $0.65_{-0.12}^{+0.14}$ expected for the LMA solution [10]. The predicted rates are $80.6 \pm 2.6 \mathrm{SNU}$ for the Ga experiments and $2.69 \pm 0.31 \mathrm{SNU}$ for the $\mathrm{Cl}$ experiment. The corresponding $\chi^{2}$ for the rates fit is 3.4 for 4 d.o.f.

In Fig. 4 we show the survival probability for a neutrino produced in the center of the Sun as a function of the neutrino energy, taking $\Delta m^{2}=2.5 \times 10^{-6} \mathrm{eV}^{2}$. The three curves correspond to three different normalizations of the magnetic field eigenmode, $B_{\max }=0.45,0.5,0.55$ MG. We caution the reader that the flux of the $p p$ neu-

\footnotetext{
${ }^{\ddagger}$ Fortunately, in the case of the lowest field eigenmode a considerable simplification occurs because of the $\sin \Theta$ dependence of the field on the polar angle. Still, a 2-dimensional numerical integration is required.
}

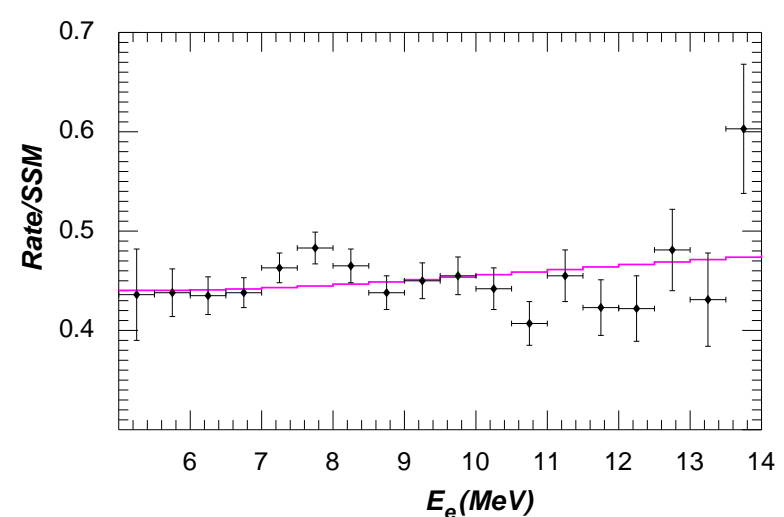

FIG. 5. Predicted SuperKamiokande recoil electron energy spectrum for $B_{\max }=0.50 \mathrm{MG}$ and $\Delta m^{2}=2.5 \times 10^{-6} \mathrm{eV}^{2}$.

trinos cannot be easily read off from this plot, since, as already mentioned, to compute it one has to appropriately integrate over the production region.

The survival probability is not strictly constant for high energy ${ }^{8} \mathrm{~B}$ neutrinos, however, its variation with energy is rather small. The recoil electron energy spectrum expected at SK is shown in Fig. 5. The value of the $\chi^{2}$ is 24 for $19 \mathrm{~d}$. o. f. The spectrum becomes flatter as one increases $\Delta m^{2}$.

It is worth stressing that the value of the field that gives a good fit trivially scales with the value of the transition moment according to $B_{\max }^{\prime}=B_{\max } \times 10^{-11} \mu_{B} / \mu_{e \mu}$. In particular, if one chooses $\mu_{e \mu}=3 \times 10^{-12} \mu_{B}$ in order to satisfy the astrophysical bound given in [20], one can choose $B_{\max }^{\prime} \sim 1.5-2 \mathrm{MG}$, which is still perfectly acceptable, as shown in [30]. No property of the field, such as its lifetime, etc., is affected by such a rescaling.

Our scenario may be convincingly tested (and perhaps excluded) by the KamLAND experiment, should it establish flavor oscillations with the LMA parameters, or by the Borexino experiment, should it observe large daynight variations, as predicted for the LOW solution (SFF predicts no variation).

There are three potential sources of time variations in our scenario. First, the effect of the time varying $\mathrm{CZ}$ field can be shown to be negligibly small, assuming $B_{C Z} \lesssim 100$ $\mathrm{kG}$ and $B_{R Z}$ and $\Delta m^{2}$ in the range of the fit. Second, if the field is not axially symmetric, one expects 27 -day variations. Third, if, in addition to $l=1$ modes, the $l=2$ modes $\left(P_{2}^{1}(\cos \Theta) \propto \sin 2 \Theta\right)$ are also present, the flux may exhibit annual variations, for the same reason that semiannual variations are expected for the CZ SFF. These variations have extrema in March and September, and hence are distinguishable from the variations due to the eccentricity of the Earth's orbit. Calculations show that for the points in the allowed region a relative change in the SK rate is $\delta R / R \sim 2 \delta B / B$. Hence, even if the $l=2$ and $l=1$ modes have comparable amplitudes, the rate 
variation is expected to be of the order of a few percent, in contrast to SFF in the CZ, in which case it is expected to be much larger. The current data provide a weak upper bound on the strength of the $l=2$ modes. Both the 27day and the annual variations described above constitute smoking gun signatures of the RZ SFF mechanism.

In summary, our scenario provides a fit to all available solar neutrino data, is compatible with all existing constraints, and may be tested in the very near future.

\section{ACKNOWLEDGMENTS}

We would like to thank John Bahcall, Sarbani Basu, Axel Brandenburg, Gia Dvali, and Cecilia Lunardini for useful discussions. A. F. was supported by the W. M. Keck Foundation.

[1] Q. R. Ahmad et al., Phys. Rev. Lett. 87, 071301 (2001).

[2] Q. R. Ahmad et al. [SNO Collaboration], Phys. Rev. Lett. 89, 011301 (2002) [arXiv:nucl-ex/0204008].

[3] S. Fukuda et al., Phys. Rev. Lett. 86, 5651 (2001); Phys. Rev. Lett. 86, 5656 (2001).

[4] S. Fukuda et al. [Super-Kamiokande Collaboration], Phys. Lett. B 539, 179 (2002) [arXiv:hep-ex/0205075].

[5] C. Giunti, Phys. Rev. D 65, 033006 (2002); J. Bahcall, Phys. Rev. C 65, 015802 (2002); V. Berezinsky, Astropart. Phys. 17, 509 (2002) [arXiv:hep-ph/0108166].

[6] The absence of the inverse $\beta$-decay signal in the SuperKamiokande data provides a strong limit on the possible flux of electron antineutrinos from the Sun. The limit, based on the first 825 days of Super-Kamiokande running, is $\Phi_{\bar{\nu}_{e}}\left({ }^{8} B\right) \lesssim 1.8 \times 10^{5} \mathrm{~cm}^{-2} \mathrm{~s}^{-1}$, or $\lesssim 3.4 \%$ of the Standard Solar Model prediction; see P. Vogel and J. F. Beacom, Phys. Rev. D 60, 053003 (1999) [arXiv:hep-ph/9903554]; E. Torrente-Lujan, Phys. Lett. B 494, 255 (2000) [arXiv:hep-ph/9911458]. The possibility to observe the solar electron antineutrinos at SNO was studied in A. B. Balantekin and F. Loreti, Phys. Rev. D 45, 1059 (1992).

[7] S. Nakamura, T. Sato, S. Ando, T. S. Park, F. Myhrer, V. Gudkov and K. Kubodera, Nucl. Phys. A 707, 561 (2002) [arXiv:nucl-th/0201062].

[8] J. N. Bahcall, E. Lisi, D. E. Alburger, L. De Braeckeleer, S. J. Freedman and J. Napolitano, Phys. Rev. C 54, 411 (1996) [arXiv:nucl-th/9601044].

[9] J. N. Bahcall, M. H. Pinsonneault and S. Basu, Astrophys. J. 555, 990 (2001) [arXiv:astro-ph/0010346].

[10] J. N. Bahcall, M. C. Gonzalez-Garcia and C. Pena-Garay, JHEP 0204, 007 (2002) [arXiv:hep-ph/0111150].

[11] V. N. Gribov and B. Pontecorvo, Phys. Lett. B 28, 493 (1969); L. Wolfenstein, Phys. Rev. D17, 2369 (1978); S. P. Mikheev and A. Y. Smirnov, Yad. Fiz. (Sov. J.
Nucl. Phys.) 42, 913 (1985); S. P. Mikheev and A. Y. Smirnov, Nuovo Cim. 9C, 17 (1986).

[12] A. Cisneros, Astrophys. Space Sci. 10, 87 (1971).

[13] M. B. Voloshin and M. I. Vysotsky, L. B. Okun, Sov. Phys. JETP64, 446 (1986).

[14] E. K. Akhmedov, Phys. Lett. B 213, 64 (1988).

[15] C. Lim and W. Marciano, Phys. Rev. D 37, 1368 (1988).

[16] R. S. Raghavan, A. B. Balantekin, F. Loreti, A. J. Baltz, S. Pakvasa and J. Pantaleone, Phys. Rev. D 44, 3786 (1991).

[17] J. Schechter and J. W. Valle, Phys. Rev. D 24, 1883 (1981) [Erratum-ibid. D 25, 283 (1981)].

[18] D. A. Krakauer et al., Phys. Lett. B 252, 177 (1990).

[19] A. I. Derbin, A. V. Chernyi, L. A. Popeko, V. N. Muratova, G. A. Shishkina and S. I. Bakhlanov, JETP Lett. 57, 768 (1993) [Pisma Zh. Eksp. Teor. Fiz. 57, 755 (1993)].

[20] G. G. Raffelt, Phys. Rev. Lett. 64, 2856 (1990); G. G. Raffelt, Phys. Rept. 320, 319 (1999).

[21] Achim Weiss, private communication.

[22] PDG, Eur. Phys. J. C 15 (2000); http://pdg.lbl.gov.

[23] E. N. Parker in The Structure of the Sun, Proc. of the VI Canary Islands School, Cambridge U. Press 1996, p. 299.

[24] H. M. Antia, S. M. Chitre, and M. J. Thompson, A\&A 360, 335 (2000).

[25] O. G. Miranda, C. Pena-Garay, T. I. Rashba, V. B. Semikoz and J. W. Valle, Nucl. Phys. B 595, 360 (2001) [arXiv:hep-ph/0005259]; Phys. Lett. B 521, 299 (2001) [arXiv:hep-ph/0108145]; J. Pulido, hepph/0101116; hep-ph/0106201; Astropart. Phys. 18, 173 (2002) [arXiv:hep-ph/0112104]; A. M. Gago et al., Phys. Rev. D 65, 073012 (2002) [arXiv:hep-ph/0112060].

[26] http://www.ngdc.noaa.gov/stp/SOLAR/SSN/ssn.html.

[27] J. N. Bahcall, Neutrino Astrophysics, Cambridge, UK, UNIV. PR. (1989).

[28] T. G. Cowling, MNRAS 105, 166 (1945)

[29] J. Bahcall and R. Ulrich, Astrophys. J. 170, 593 (1971)

[30] A. Friedland and A. Gruzinov, astro-ph/0211377, accepted for publication in Astrophys. J.

[31] A. Friedland, Phys. Rev. D 64, 013008 (2001); hepph/0106042.

[32] E. Bellotti [GNO Collaboration], Nucl. Phys. Proc. Suppl. 91, 44 (2001).

[33] V. N. Gavrin [SAGE Collaboration], Nucl. Phys. Proc. Suppl. 91, 36 (2001).

[34] B. Cleveland et al., Astrophys. J. 496, 505 (1998). 